\title{
A Bias-aware EnKF Estimator for Aerodynamic Flows
}

\author{
Andre F. C. da Silva* and Tim Colonius ${ }^{\dagger}$ \\ California Institute of Technology, Department of Mechanical and Civil Engineering, Pasadena, CA, 91101
}

\begin{abstract}
Ensemble methods can integrate measurement data and CFD-based models to estimate the state of fluid systems in a robust and cost-efficient way. However, discretization errors can render numerical solutions a biased representation of reality. Left unaccounted for, biased forecast and observation models can lead to poor estimator performance. In this work, we propose a low-rank representation for the bias whose dynamics is represented by a colorednoise process. System state and bias parameters are simultaneously corrected on-line with the Ensemble Kalman Filter (EnKF) algorithm. The proposed methodology is demonstrated to achieve a 70\% error reduction for the problem of estimating the state of the two-dimensional low-Re flow past a flat plate at high angle of attack using an ensemble of coarse-mesh simulations and pressure measurements at the surface of the body, compared to a bias-blind estimator. Strategies to determine the bias statistics and to deal with nonlinear observation functions in the context of ensemble methods are discussed.
\end{abstract}

\section{Nomenclature}

$x_{k}=$ system state vector at time $t_{k}$

$y_{k}=$ vector of measured quantities at time $t_{k}$

$z_{k}=$ augmented state vector at time $t_{k}$

$f(\cdot) \quad=\quad$ (possibly nonlinear) system dynamic model

$h(\cdot) \quad=$ (possibly nonlinear) observation model

$\mu_{k}, v_{k}=$ process (i.i.d. $\left.N(0, Q)\right)$ and observation (i.i.d. $\left.N(0, R)\right)$ noises

$C_{k}^{x y}=$ cross-covariance matrix between variables $x$ and $y$ at time $t_{k}$

$Z_{k} \quad=$ state ensemble matrix (each column represents the state of the corresponding ensemble member)

$A_{k} \quad=$ state perturbation matrix (see Eq. 12, at time $t_{k}$

$\mathbb{1}=1$-dimensional vector of ones

$\xi_{k}, \eta_{k}=$ respectively, dynamics and observation bias parameters

$\Gamma_{x}, \Gamma_{y}=$ respectively, dynamics and observation bias low-rank models

$v_{k} \quad=$ analysis correction coefficients

$n=$ dimension of the system state vector

$n_{o}=$ dimension of the observation bias parameters vector

$n_{s} \quad=$ dimension of the state bias parameters vector

$q=$ ensemble size

$p \quad=$ dimension of the measurement/observation vector

$\|\cdot\|_{A}=$ Weighted L2-norm defined as $\|x\|_{A}=\left\|A^{-1 / 2} x\right\|_{2}$

\section{Introduction}

Predicting the state of a fluid system and forecasting its evolution are crucial to effective closed-loop control design. The pursuit of a seamless integration of the available measurement data and often complex mathematical models in order to produce a reliable and robust estimate of the state of a dynamical system is the perennial goal of the data assimilation research community.

Most of the classical estimation techniques that have been developed within the control theory community are only computational feasible for systems with a reduced number of degrees of freedom. One possible approach to

\footnotetext{
*Graduate Student, andrefcs@caltech.edu, AIAA student member.

${ }^{\dagger}$ Frank and Ora Lee Marble Professor of Mechanical Engineering, AIAA Associate Fellow.
} 
circumvent this issue is by using dimensionality reduction techniques such as Balanced Truncation[1] or Eigenvalue Realization Algorithm [2] to highlight the most important features of the dynamical system under scrutiny. The resulting reduced-order models (ROM) can be made small enough to allow the use of the standard algorithms, but their well-known fragility to the specification of initial conditions and flow parameters (e.g., Reynolds number) can constitute a major applicability limitation.

On the other hand, researchers in areas such as meteorology and geophysics were lead to develop estimation algorithms that are capable of handling high-dimensional models and large volumes of data. Recently, some of these techniques have been applied to faster, engineering-scale flows. Colburn et al. [3] applied a Ensemble Kalman Filter (EnKF) to the problem of estimating the statistics of the 3D turbulent channel flow. Kikuchi et al. 4] compared the performance of a EnKF and a Particle Filter applied to POD-Galerkin model of the problem of the flow past a cylinder. Kato et al. [5] used a variation of the EnKF to achieve synchronization between a RANS-SA numerical simulation of a steady transonic flow past airfoils and pressure experimental data. Mons et al. [6] used a Kalman smoother and other variational methods to reconstruct freestream perturbation history based on measurements taken on and around a circular cylinder subjected to it. da Silva and Colonius [7] used an EnKF-based estimator to estimate free-stream perturbations from pressure measurements taken on the surface of a NACA 0009 airfoil at high angle of attack.

These efforts notwithstanding, the success of the estimator is contingent on the accuracy of the model chosen to represent the dynamics. Left untreated, forecast and measurement bias can lead the estimator to converge to the wrong solution or become unstable. Friedland [8] was one of the first to propose a direct treatment of the forecast error. He proposed a two-stage sequential estimator in which state and bias vector were treated independently, termed the Separate-bias Kalman Filters (SepKF). Later, Dee and Da Silva [9] built upon previous work to deliver a rigorous method to independently estimate and sequentially correct for forecast bias. Drecourt et al. [10] compared this method to the colored-noise Kalman Filter (ColKF), in which the state vector is augmented to account for noise processes modeled by autoregressive models. These works, however, all assume that the observation model is unbiased.

When the forecast and observation models involve the temporal and spatial discretization of a set of partial differential equations such as the Navier-Stokes equations, the resolution error is an additional source of bias that can affect both dynamics and the observation models. These errors can be particularly harmful in the context of ensemble methods when the ensemble size is much smaller than the number of degrees of freedom of the forecast model. Since the corrections applied to forecast state lie in the low-dimensional subspace spanned by the prior ensemble perturbations, large dynamic bias can render the true state of the system unreachable.

The observation model may be responsible for the appearance of two additional kinds of error. The first also emerges from the discretization process and leads to problems that are similar to the ones that would be encountered if the assimilated measurements displayed a systematic error, with the complication that this error may now be state dependent. The second error source comes from the filtering itself and only appears when the observation model is nonlinear. Even though the standard EnKF theory was laid down for strictly linear observation functions, extensions inspired by the extended Kalman filter and the iterated Kalman filter [11] were proposed to allow for measurements that relate to the state through a nonlinear map[12, 13]. That notwithstanding, the fact that, in this case, the posterior distribution may no longer be Gaussian may render the analysis itself biased.

In this work, a direct treatment of both discretization bias sources is proposed. In section III it is demonstrated that low-resolution models gives rise to biased estimates. In section IV we model these errors by representing the most dynamically relevant features of the bias as colored-noise processes. The proposed bias-aware methodology is summarized in section $\mathrm{V}$ Section $\mathrm{VI}$ describes the numerical experiments used to assess the performance and section VII list some of the main conclusions of this work and proposes future research directions.

\section{Resolution Error as a Source of Bias}

Let $\tilde{f}(x)$ be the exact transition function of the Navier-Stokes equations, which maps the infinite-dimensional solution $\tilde{x}_{k-1}$ at time $t_{k-1}$ to the solution $\tilde{x}_{k}$ at time $t_{k}$,

$$
\begin{aligned}
& \tilde{x}_{k}=\tilde{f}_{k}\left(\tilde{x}_{k-1}\right), \\
& y_{k}=\tilde{h}\left(\tilde{x}_{k}\right)+\epsilon_{k}^{m},
\end{aligned}
$$

where $\epsilon_{k}^{m} \sim N\left(0, \tilde{R}_{k}\right)$ is a $m$-dimensional random error vector that is independent of the state and uncorrelated in time, being usually related to the measurement methodology. Since both the state $\tilde{x}$ and the operators $\tilde{f}$ and $\tilde{h}$ are unattainable for practical purposes, we introduce a finite-dimensional approximation for the model and state. We notate 
the finite-dimensional approximations with the same symbols but without the tilde.

Following Cohn[14], we define a projection operator $\Pi$ that maps the the true state $\tilde{x}_{k}$ onto its finite-dimensional representation $x_{k}=\Pi \tilde{x}_{k}$. The propagation of $x_{k}$ can be represented as

$$
x_{k}=f\left(x_{k-1}\right)+\delta_{k-1},
$$

where

$$
\delta_{k}=\Pi \tilde{f}\left(\tilde{x}_{k}\right)-f\left(\Pi \tilde{x}_{k}\right) .
$$

The forcing term $\delta_{k}$ represents the model error, and gathers errors from different sources: discretization error, inaccurate boundary conditions, uncertain forcing, and so on.

Analogously, since the continuous state is never available for practical purposes, a discrete version of the observation operator $h(\cdot)$ needs to be introduced:

$$
y_{k}=h_{k}\left(x_{k}\right)+\epsilon_{k}^{m}+\epsilon_{k}^{r},
$$

where

$$
\begin{aligned}
\epsilon_{k}^{r} & =\tilde{h}\left(\tilde{x}_{k}\right)-h\left(\Pi \tilde{x}_{k}\right) \\
& =\left(\tilde{h}\left(\tilde{x}_{k}\right)-\tilde{h}\left(\Pi \tilde{x}_{k}\right)\right)+\left(\tilde{h}\left(\Pi \tilde{x}_{k}\right)-h\left(\Pi \tilde{x}_{k}\right)\right) .
\end{aligned}
$$

is the error of representativeness [14]. This error can be further split into two contributions: a first term that represents the effect of the exact operator on the unresolved scales, and a second term that represents the discretization error of the operator itself.

\section{Low-rank Representation of the Bias}

Because $\delta_{k}$ depends not only on the state but also on the continuous operator $\tilde{f}$, its value is unknowable from a deterministic point of view. Therefore, the most common approach is to represent this error as a stochastic perturbation with known bias and covariance. Thus, we use

$$
\delta_{k}=\Gamma_{x} \xi_{k}+\mu_{k}
$$

where $\Gamma_{x}$ is a low-rank representation of the low-frequency behavior of the bias and represents the available deterministic knowledge about the model error. The second term $\left(\mu_{k} \sim N\left(0, Q_{k}\right)\right)$ represents the high-frequency portion of the bias, and it is usually regarded as being white.

A complete representation of $\delta_{k}$ requires an impractical amount of data and operations. Since the dimension $p$ of the measurement vector obtained at any given time is customarily smaller than the number $n$ of degrees of freedom of the forecast model, it is not possible to estimate all $n(n+1) / 2$ degrees of freedom of the covariance matrix associated to $\delta_{k}$ in real time, regardless of the estimation procedure used [15].

The measurement resolution error can also be split in two terms:

$$
\epsilon_{k}^{r}=\Gamma_{y} \eta_{k}+v_{k},
$$

where $\Gamma_{y}$ is a low-rank representation of the low-frequency behavior of the bias, and $v_{k} \sim N\left(0, R^{r}\right)$ represents the high-frequency content of the error in the measurement error. The measurement accuracy error $\epsilon_{k}^{m}$ is merged to $v_{k}$, which represent the zero-mean high-frequency portion of the observation error, which is now represented as zero-mean Gaussian process with a combined covariance matrix $R$.

Due to its very nature, it is to be expected that $\xi_{k}$ and $\eta_{k}$ are auto-correlated in time. In the discrete-time framework, autoregressive models (AR) are the simplest way of representing this feature. Noise sequences obeying AR models are often referred to as colored-noise sequences [10, 16]. Assuming the Markov condition holds, their dynamics are given by

$$
\begin{aligned}
\xi_{k} & =\bar{\xi}+\Phi_{x}\left(\xi_{k-1}-\bar{\xi}\right)+\gamma_{x, k} \\
\eta_{k} & =\bar{\eta}+\Phi_{y}\left(\eta_{k-1}-\bar{\eta}\right)+\gamma_{y, k}
\end{aligned}
$$


where $\gamma_{x, k} \sim N\left(0, Q^{b}\right)$ and $\gamma_{y, k} \sim N\left(0, R^{b}\right)$, and $\Phi_{x}$ and $\Phi_{y}$ should be determined based on prior knowledge about the system under study. For example, if these error terms are supposed to vary in a much slower time scale than the state dynamics, one can choose to use a persistent model and set $\Phi_{x}=\Phi_{y}=1$.

In order to take advantage of an eventual nonzero cross-correlation between the bias parameters $\xi$ and $\eta$ and the state $x$ in the filtering process, these variables are gathered in an augmented state vector $\left(z=\left[x^{T} \xi^{T} \eta^{T}\right]^{T}\right)$ which will be estimated using the algorithm proposed in the next section.

\section{A Bias-aware Ensemble Kalman Filter}

The Ensemble Kalman Filter is an estimation technique proposed by Evensen[17] in 1994 with the goal of overcoming the prohibitive cost involved with the state covariance propagation for standard techniques such as the classical Kalman Filter (KF) or its nonlinear generalizations (Extended Kalman Filter or the Unscented Kalman Filter[18]). It can be understood as a Monte Carlo approximation to the KF: the internal state of the estimator is represented by an ensemble of particles so that the corresponding ensemble mean $\bar{z}$ and covariance matrix $C^{z z}$ are used to approximate their population counterparts.

In a discrete setting, the filtering process can be summarized in two main steps:

Forecast Step: The state of each ensemble member (here denoted by the superscript $(j)$ ) at the next time step is forecast using the (possibly nonlinear) dynamic model:

$$
\begin{aligned}
\hat{z}_{k}^{(j)} & =\left[\begin{array}{c}
\hat{x}_{k}^{(j)} \\
\hat{\xi}_{k}^{(j)} \\
\hat{\eta}_{k}^{(j)}
\end{array}\right]=f^{\dagger}\left(z_{k-1}^{(j)}\right)+\mu_{k}^{\dagger(j)} \\
& =\left[\begin{array}{c}
f\left(x_{k-1}^{(j)}\right) \\
\bar{\xi}+\Phi_{x}\left(\xi_{k-1}^{(j)}-\bar{\xi}\right) \\
\bar{\eta}+\Phi_{y}\left(\eta_{k-1}^{(j)}-\bar{\eta}\right)
\end{array}\right]+\left[\begin{array}{c}
\mu_{k}^{(j)} \\
\gamma_{x, k}^{(j)} \\
\gamma_{y, k}^{(j)}
\end{array}\right]
\end{aligned}
$$

If applied to a linear system, the ensemble approach reduces the cost associated with the time-propagation of the covariance matrix from $O\left(n^{3}\right)$ for the classical KF to $O\left(n^{2} q\right)$ for the EnKF, assuming $n_{s} \ll n$ and $n_{o} \ll n$.

The prior statistics are defined as

$$
\begin{aligned}
\hat{Z}_{k} & =\left[\begin{array}{llll}
\hat{z}_{k}^{(1)} & \hat{z}_{k}^{(2)} & \ldots & \hat{z}_{k}^{(q)}
\end{array}\right] \\
\bar{z}_{k} & =\frac{1}{q} \hat{Z}_{k} \mathbb{1} \\
\hat{C}_{k}^{z z} & =\frac{1}{\sqrt{q-1}} \sum_{i=1}^{q}\left(\hat{z}_{k}^{(i)}-\bar{z}_{k}\right)\left(\hat{z}_{k}^{(i)}-\bar{z}_{k}\right)^{T}
\end{aligned}
$$

which can also be expressed in terms of the scaled ensemble perturbation matrix

$$
\begin{aligned}
\hat{A}_{k} & =\frac{1}{\sqrt{q-1}} \hat{Z}_{k}\left(I-\bar{z}_{k} \mathbb{1}^{T}\right) \\
\hat{C}_{k}^{z z} & =\hat{A}^{(j)}\left(\hat{A}^{(j)}\right)^{T}
\end{aligned}
$$

Analysis Step: Bayes theorem can be used to combine the probability density function (PDF) of forecast state (often referred to as the prior distribution) with newly-available measurement statistics to produce the PDF of the analyzed state (often referred to as the posterior distribution). The formal solution to the Kalman filtering problem is defined as the state that minimizes the conditional expectation of the mean-square error, i.e., the mean of the posterior distribution [11].

There are several alternative ways of interpreting this definition. Particularly instructive in the context of the ensemble methods is to frame it as an optimization problem. For each of the ensemble members, provided that both the prior and 
the measurement distributions are Gaussian, the mode (maximum-likelihood estimate) of the posterior distribution corresponds to the minimizer of the cost function:

$$
J(z)=\frac{1}{2 \alpha}\left\|G^{-1} z-\hat{z}_{k}^{(j)}\right\|_{\hat{C}_{k}^{z z}}^{2}+\frac{1}{2}\left\|y_{k}-h(x)-\Gamma_{y} \eta-v_{k}^{(j)}\right\|_{R}^{2}
$$

while restricting $\left[\begin{array}{ccc}x-\Gamma_{x} \xi & \xi & \eta\end{array}\right]^{T}=G^{-1} z$ to the affine subset generated by the prior estimate of the state $\hat{z}_{k}^{(j)}$ and the subspace spanned by the scaled perturbation matrix $\hat{A}_{k}$, i. e.,

$$
z_{k}=\underset{z \in G\left(\hat{z}+\hat{A}_{k}\right)}{\operatorname{argmin}} J(z)
$$

where

$$
G=\left[\begin{array}{ccc}
I & \Gamma_{x} & 0 \\
0 & I & 0 \\
0 & 0 & I
\end{array}\right]
$$

The first term in the cost function acts as an regularization term by penalizing the distance to prior estimated by the proposed dynamical model, and the second term penalizes the data mismatch between the observed measurement $y_{k}$ and the ones predicted by the proposed observation model. The relative reliability of these two models is prescribed by the matrices $R$ (measurement noise covariance matrix) and $\hat{C}_{k}$ (prior ensemble state covariance). Here, the parameter $\alpha$ represents the magnitude of the multiplicative covariance inflation. If, for example, the observation function is linear, the posterior is guaranteed to remain Gaussian and, the maximum-likelihood estimate (posterior mode) is also the minimum-variance estimate (posterior mean).

When finite ensemble sizes are used, EnKF analysis systematically underestimates error covariances[19]. Left unattended, this fact can lead to covariance collapse, where each ensemble member predicts the same (possibly incorrect) dynamics. The most common empirical algorithm fix is covariance inflation (CI). This technique artificially increases the ensemble covariance in order to weight the measurement data more heavily. In general, the covariance inflation can be implemented as

$$
\hat{z}^{(j)}=\bar{z}+\alpha\left(\hat{z}^{(j)}-\bar{z}\right)+\beta^{(j)},
$$

where $\beta^{(j)}$ is the additive covariance inflation vector that is usually drawn from a zero-mean normal distribution with a predefined covariance matrix, and $\alpha$ is the multiplicative covariance inflation parameter. Multiplicative $\mathrm{CI}$ is used to correct the filter transient behavior by delaying the collapse of the covariance, while additive CI will enforce a lower bound to the system covariance, limiting its perceived reliability. The simplest multiplicative inflation scheme[20] assumes $\alpha$ in Eq. [16 to be a scalar, but more sophisticated schemes are also available[21].

\section{A. The Linear Observation Function Case}

When the observation function is linear, i.e. $h(x)=H x$, the observation equation can be written as

$$
\hat{y}_{k}^{(j)}=\tilde{H} G \hat{z}_{k}^{(j)}+v_{k}^{(j)}
$$

where

$$
\tilde{H}=\left[\begin{array}{lll}
H & 0 & \Gamma_{y}
\end{array}\right]
$$

The restriction on the optimization space is enforced by means of a change of variables

$$
z=G\left(\hat{z}_{k}^{(j)}+\sqrt{\alpha} \hat{A}_{k} v\right)
$$

where $v \in \mathbb{R}^{q}$ is the correction coefficient vector.

The analysis step objective is then defined as finding

$$
v_{k}=\underset{v \in \mathbb{R}^{q}}{\operatorname{argmin}} J(v)
$$

where

$$
J(v)=\frac{1}{2}\|v\|^{2}+\frac{1}{2}\left\|y_{k}-\tilde{H} G\left(\hat{z}_{k}^{(j)}+\sqrt{\alpha} \hat{A}_{k} v\right)-v_{k}^{(j)}\right\|_{R}^{2}
$$


Since $J(v)$ is quadratic in $v$, the solution is unique and corresponds to the root of

$$
D J(v)=v-\sqrt{\alpha}\left(\tilde{H} G \hat{A}_{k}\right)^{T} R^{-1}\left(y_{k}-\tilde{H} G \hat{z}_{k}^{(j)}-\sqrt{\alpha} \tilde{H} G \hat{A}_{k} v-v_{k}^{(j)}\right)=0
$$

which is given by

$$
\begin{aligned}
v_{k}^{(j)} & =\sqrt{\alpha}\left[I+\alpha\left(\tilde{H} G \hat{A}_{k}\right)^{T} R^{-1}\left(\tilde{H} G \hat{A}_{k}\right)\right]^{-1}\left(\tilde{H} G \hat{A}_{k}\right)^{T} R^{-1}\left(y_{k}-\tilde{H} G \hat{z}_{k}^{(j)}-v_{k}^{(j)}\right) \\
& =\sqrt{\alpha}\left(\tilde{H} G \hat{A}_{k}\right)^{T}\left[R+\alpha\left(\tilde{H} G \hat{A}_{k}\right)\left(\tilde{H} G \hat{A}_{k}\right)^{T}\right]^{-1}\left(y_{k}-\tilde{H} G \hat{z}_{k}^{(j)}-v_{k}^{(j)}\right)
\end{aligned}
$$

where we have used the Woodbury matrix identity to obtain the second line.

Notice that here we have the possibility to choose between performing the analysis in the ensemble space ( $q$-by- $q$ matrix inversion - Eq. 23a), or in the measurement space ( $p$-by- $p$ matrix inversion - Eq. 23b), depending on which one is smaller.

The final solution is then obtained by projecting these coefficients back to the state space:

$$
\begin{aligned}
z_{k}^{(j)} & =G \hat{z}_{k}^{(j)}+\alpha G \hat{A}_{k}\left[I+\alpha\left(\tilde{H} G \hat{A}_{k}\right)^{T} R^{-1}\left(\tilde{H} G \hat{A}_{k}\right)\right]^{-1}\left(\tilde{H} G \hat{A}_{k}\right)^{T} R^{-1}\left(y_{k}-\tilde{H} G \hat{z}_{k}^{(j)}-v_{k}^{(j)}\right) \\
& =G \hat{z}_{k}^{(j)}+\alpha G \hat{A}_{k}\left(\tilde{H} G \hat{A}_{k}\right)^{T}\left[R+\alpha\left(\tilde{H} G \hat{A}_{k}\right)\left(\tilde{H} G \hat{A}_{k}\right)^{T}\right]^{-1}\left(y_{k}-\tilde{H} G \hat{z}_{k}^{(j)}-v_{k}^{(j)}\right)
\end{aligned}
$$

Algorithmically, when the inversion is done in the measurement space, instead of solving for $v_{k}$, the representers formulation [22] is used

$$
\begin{array}{r}
{\left[R+\alpha\left(\tilde{H} G \hat{A}_{k}\right)\left(\tilde{H} G \hat{A}_{k}\right)^{T}\right] b_{k}^{(j)}=y_{k}-\tilde{H} G \hat{z}_{k}^{(j)}-v_{k}^{(j)}} \\
z_{k}^{(j)}=G \hat{z}_{k}^{(j)}+\alpha G \hat{A}_{k}\left(\tilde{H} G \hat{A}_{k}\right)^{T} b_{k}^{(j)}
\end{array}
$$

where the columns of $\alpha G \hat{A}_{k}\left(\tilde{H} G \hat{A}_{k}\right)^{T}$ are known as the representers. They correspond to the influence fields of each of the measurement locations onto the corrected solution, and can be used to provide a posteriori advisement on optimal sensor placement[7].

\section{B. The Nonlinear Observation Function Case}

When $h(x)$ is nonlinear, $J(z)$ is no longer quadratic, and may neither be convex nor have a single minimum. Furthermore, as the Jacobian $H(x)=\frac{\partial h}{\partial x}(x)$ is now state dependent, Eq. 24 can not be used to directly compute the minimizer of the cost function. In fact, the maximum likelihood estimate (the conditional mode of the state) may not coincide with the minimum mean square error estimate (the conditional expectation of the state), which is the formal solution of the Kalman filtering problem.

Several approaches that have been devised to deal with the nonlinear case are discussed in the following sections.

\section{Implicit Linearization}

This approach was originally proposed by Evensen [23]. As discussed in appendix A, this approach can be understood as an approximation to an extended Kalman filter in which the observation function is linearized about the ensemble mean. Since the linearized operator is never explicitly computed, we use the expression implicit linearization to refer to this scheme. We again start by augmenting the state vector with the predicted measurements. The new 
observation function simply selects the last variable of the state vector and is, therefore, linear.

$$
\begin{aligned}
& \hat{w}_{k}=\left[\begin{array}{c}
\hat{x}_{k} \\
\hat{\xi}_{k} \\
\hat{\eta}_{k} \\
\hat{y}_{k}
\end{array}\right]=f^{\dagger}\left(w_{k-1}\right)+\mu_{k}^{\dagger} \\
& =\left[\begin{array}{c}
f\left(x_{k-1}\right) \\
\Phi_{x} \xi_{k-1}+\left(I-\Phi_{x}\right) \bar{\xi} \\
\Phi_{y} \eta_{k-1}+\left(I-\Phi_{y}\right) \bar{\eta} \\
h\left(f\left(x_{k-1}\right)+\Gamma_{x} \xi_{k}\right)+\Gamma_{y} \eta_{k}
\end{array}\right]+\left[\begin{array}{c}
\mu_{k} \\
\gamma_{x, k} \\
\gamma_{y, k} \\
v_{k}
\end{array}\right] \\
& \hat{y}_{k}=\left[\begin{array}{llll}
0 & 0 & 0 & I
\end{array}\right] \hat{w}_{k}=L \hat{w}_{k}
\end{aligned}
$$

The associated cost function is

$$
J(w)=\frac{1}{2 \alpha}\left\|\tilde{G}^{-1} w-\hat{w}_{k}\right\|_{\hat{C}_{k}^{w w}}^{2}+\frac{1}{2}\left\|y_{k}-L w\right\|_{R}^{2}
$$

where

$$
\tilde{G}=\left[\begin{array}{ll}
G & 0 \\
0 & I
\end{array}\right]
$$

and

$$
\begin{aligned}
& \hat{C}_{k}^{w w}=\left[\begin{array}{cc}
\hat{C}_{k}^{z z} & \hat{C}_{k}^{z y} \\
\left(\hat{C}_{k}^{z y}\right)^{T} & \hat{C}_{k}^{y y}
\end{array}\right]=\left[\begin{array}{cccc}
\hat{C}_{k}^{x x} & \hat{C}_{k}^{x \xi} & \hat{C}_{k}^{x \eta} & \hat{C}_{k}^{x y} \\
\left(\hat{C}_{k}^{x \xi}\right)^{T} & \hat{C}_{k}^{\xi \xi} & \hat{C}_{k}^{\xi \eta} & \hat{C}_{k}^{\xi y} \\
\left(\hat{C}_{k}^{x \eta}\right)^{T} & \left(\hat{C}_{k}^{\xi \eta}\right)^{T} & \hat{C}_{k}^{\eta \eta} & \hat{C}_{k}^{\eta y} \\
\left(\hat{C}_{k}^{x y}\right)^{T} & \left(\hat{C}_{k}^{\xi y}\right)^{T} & \left(\hat{C}_{k}^{\eta y}\right)^{T} & \hat{C}_{k}^{y y}
\end{array}\right] \\
& =\frac{1}{q-1} \sum_{j=1}^{q}\left(\hat{w}_{k}^{j}-\bar{w}_{k}\right)\left(\hat{w}_{k}^{j}-\bar{w}_{k}\right)^{T} \\
& =\hat{A}_{k}^{w}\left(\hat{A}_{k}^{w}\right)^{T}
\end{aligned}
$$

Using a change of variables similar to the one proposed in Eq. 19, Eq. 28 can be rewritten as

$$
\begin{gathered}
w=\tilde{G}\left(\hat{w}_{k}^{(j)}+\sqrt{\alpha} \hat{A}_{k}^{w} v\right) \\
J(v)=\frac{1}{2}\|v\|^{2}+\frac{1}{2}\left\|y_{k}-L \tilde{G}\left(\hat{z}_{k}^{(j)}+\sqrt{\alpha} \hat{A}_{k} v\right)\right\|_{R}^{2}
\end{gathered}
$$

Since this function is quadratic in $v$, the minimizer is given by

$$
\begin{aligned}
v_{k}^{(j)} & =\sqrt{\alpha}\left[I+\alpha\left(L \tilde{G} \hat{A}_{k}^{w}\right)^{T} R^{-1}\left(L \tilde{G} \hat{A}_{k}^{w}\right)\right]^{-1}\left(L \tilde{G} \hat{A}_{k}^{w}\right)^{T} R^{-1}\left(y_{k}-L \tilde{G} \hat{w}_{k}^{(j)}\right) \\
& =\sqrt{\alpha}\left(L \tilde{G} \hat{A}_{k}^{w}\right)^{T}\left[R+\alpha\left(L \tilde{G} \hat{A}_{k}^{w}\right)\left(L \tilde{G} \hat{A}_{k}^{w}\right)^{T}\right]^{-1}\left(y_{k}-L \tilde{G} \hat{w}_{k}^{(j)}\right)
\end{aligned}
$$

where

$$
\begin{gathered}
L \tilde{G} \hat{w}_{k}^{(j)}=\hat{y}_{k}^{(j)}=h\left(f\left(\hat{x}_{k-1}^{(j)}\right)+\Gamma_{x} \hat{\xi}_{k}^{(j)}\right)+\Gamma_{y} \hat{\eta}_{k}^{(j)}+v_{k}^{(j)}=h^{\dagger}\left(G \hat{z}_{k}^{(j)}\right)+v_{k}^{(j)} \\
\left(L \tilde{G} \hat{A}_{k}^{w}\right)\left(L \tilde{G} \hat{A}_{k}^{w}\right)^{T}=\hat{C}_{k}^{y y}
\end{gathered}
$$


The posterior solution is then given by

$$
\begin{aligned}
w_{k}^{(j)} & =\tilde{G} \hat{w}_{k}^{(j)}+\alpha \tilde{G} \hat{A}_{k}^{w}\left[I+\alpha\left(L \tilde{G} \hat{A}_{k}^{w}\right)^{T} R^{-1}\left(L \tilde{G} \hat{A}_{k}^{w}\right)\right]^{-1}\left(L \tilde{G} \hat{A}_{k}^{w}\right)^{T} R^{-1}\left(y_{k}-L \tilde{G} \hat{w}_{k}^{(j)}\right) \\
& =\tilde{G} \hat{w}_{k}^{(j)}+\alpha \tilde{G} \hat{A}_{k}^{w}\left(L \tilde{G} \hat{A}_{k}^{w}\right)^{T}\left[R+\alpha\left(L \tilde{G} \hat{A}_{k}^{w}\right)\left(L \tilde{G} \hat{A}_{k}^{w}\right)^{T}\right]^{-1}\left(y_{k}-L \tilde{G} \hat{w}_{k}^{(j)}\right)
\end{aligned}
$$

This approach works well as long as $h(x)$ is a monotonic function of the state (at least locally around the ensemble mean) and is not strongly nonlinear[17]. The residual $\left\|L w_{k}-h\left(x_{k}\right)-\Gamma_{y} \eta_{k}\right\|_{2}$, i.e. the difference between the analyzed

measurement to the observation operator applied to the posterior state, is a measure of the approximation introduced by this algorithm, as this quantity is expected to be zero when linear observation functions are employed.

\section{Iterative optimization}

In this approach, the posterior mean is approximated by the posterior mode, which is obtained by directly minimizing

$$
J(v)=\frac{1}{2}\|v\|^{2}+\frac{1}{2}\left\|y_{k}-h^{\dagger}\left(G\left(\hat{z}_{k}^{(j)}+\sqrt{\alpha} \hat{A}_{k} v\right)\right)-v_{k}^{(j)}\right\|_{R}^{2}
$$

by using an iterative method to find the root of

$$
D J(v)=v-\sqrt{\alpha}\left[\frac{\partial h^{\dagger}}{\partial z}\left(G\left(\hat{z}_{k}^{(j)}+\sqrt{\alpha} \hat{A}_{k} v\right)\right) G \hat{A}_{k}\right]^{T} R^{-1}\left(y_{k}-h^{\dagger}\left(G\left(\hat{z}_{k}^{(j)}+\sqrt{\alpha} \hat{A}_{k} v\right)\right)-v_{k}^{(j)}\right)=0
$$

This task can be accomplished by employing the Newton-Raphson scheme, through which successive approximations to the roots are obtained by solving

$$
\begin{aligned}
D^{2} J\left(v^{i}\right) \Delta v^{i} & =-D J\left(v^{i}\right) \\
v^{i+1} & =v^{i}+\Delta v^{i}
\end{aligned}
$$

The exact evaluation of $D^{2} J$, which requires the evaluation of $\partial^{2} h^{\dagger} / \partial z^{2}$, is often nontrivial and practically unfeasible. Since $J(v)$ is a sum of squares, we employ Gauss's approximation instead

$$
\begin{aligned}
D^{2} J(v) & \approx I+\left[\sqrt{\alpha} \frac{\partial h^{\dagger}}{\partial z}\left(G\left(\hat{z}_{k}^{(j)}+\sqrt{\alpha} \hat{A}_{k} v\right)\right) G \hat{A}_{k}\right]^{T} R^{-1}\left[\sqrt{\alpha} \frac{\partial h^{\dagger}}{\partial z}\left(G\left(\hat{z}_{k}^{(j)}+\sqrt{\alpha} \hat{A}_{k} v\right)\right) G \hat{A}_{k}\right] \\
& =I+B(v)^{T} R^{-1} B(v)
\end{aligned}
$$

in which the term with the second derivative is neglected. The resulting algorithm is known as the Gauss-Newton method. The computed increment $\Delta v$ will always be a descent direction for $J(v)$, and, if the algorithm converges, the limit will be a local extremum of $J(u)$ [24]. Due to the restriction of the optimization to the ensemble space (refer to Eq. 19), an explicit representation of $\partial h^{\dagger}(z) / \partial z$ (or its adjoint) is not required. The evaluation of $B(v)$ only requires that we know the result of applying $\partial h^{\dagger}(z) / \partial z$ to the the columns of $G \hat{A}_{k}$. If even this is not available (in the form of a linearized model, for instance), one can still estimate it using a finite difference formula

$$
\frac{\partial h^{\dagger}}{\partial z}\left(z^{*}\right) \Delta z=\frac{h^{\dagger}\left(z^{*}+\epsilon \Delta z\right)-h^{\dagger}\left(z^{*}-\epsilon \Delta z\right)}{2 \epsilon}
$$

for a sufficiently small $\epsilon$.

Substituting Eq. 40 into Eq. 39, one obtains

$$
\begin{aligned}
v^{i+1} & =v^{i}-\left(I+B\left(v^{i}\right)^{T} R^{-1} B\left(v^{i}\right)\right)^{-1}\left[v^{i}-B\left(v^{i}\right)^{T} R^{-1}\left(y_{k}-h^{\dagger}\left(G\left(\hat{z}_{k}^{(j)}+\sqrt{\alpha} \hat{A}_{k} v^{i}\right)\right)-v_{k}^{(j)}\right)\right] \\
& =\left(I+B\left(v^{i}\right)^{T} R^{-1} B\left(v^{i}\right)\right)^{-1}\left[\left(I+B\left(v^{i}\right)^{T} R^{-1} B\left(v^{i}\right)\right) v^{i}-v^{i}+B\left(v^{i}\right)^{T} R^{-1}\left(y_{k}-h^{\dagger}\left(G\left(\hat{z}_{k}^{(j)}+\sqrt{\alpha} \hat{A}_{k} v^{i}\right)\right)-v_{k}^{(j)}\right)\right] \\
& =\left(I+B\left(v^{i}\right)^{T} R^{-1} B\left(v^{i}\right)\right)^{-1} B\left(v^{i}\right)^{T} R^{-1}\left[y_{k}-h^{\dagger}\left(G\left(\hat{z}_{k}^{(j)}+\sqrt{\alpha} \hat{A}_{k} v^{i}\right)\right)+B\left(v^{i}\right) v^{i}-v_{k}^{(j)}\right] \\
& =B\left(v^{i}\right)^{T}\left(R+B\left(v^{i}\right) B\left(v^{i}\right)^{T}\right)^{-1}\left[y_{k}-h^{\dagger}\left(G\left(\hat{z}_{k}^{(j)}+\sqrt{\alpha} \hat{A}_{k} v^{i}\right)\right)+B\left(v^{i}\right) v^{i}-v_{k}^{(j)}\right]
\end{aligned}
$$


This algorithm converges in one iteration when $h(x)$ is linear, but has no convergence guarantee in the nonlinear case. In spite of that, since $\Delta v$ is a decent direction, a damped version of the algorithm can be used to ensure local convergence

$$
\begin{aligned}
v^{i+1} & =(1-\beta) v^{i}+\beta\left(I+B\left(v^{i}\right)^{T} R^{-1} B\left(v^{i}\right)\right)^{-1} B\left(v^{i}\right)^{T} R^{-1}\left[y_{k}-h^{\dagger}\left(G\left(\hat{z}_{k}^{(j)}+\sqrt{\alpha} \hat{A}_{k} v^{i}\right)\right)+B\left(v^{i}\right) v^{i}-v_{k}^{(j)}\right] \\
& =(1-\beta) v^{i}+\beta B\left(v^{i}\right)^{T}\left(R+B\left(v^{i}\right) B\left(v^{i}\right)^{T}\right)^{-1}\left[y_{k}-h^{\dagger}\left(G\left(\hat{z}_{k}^{(j)}+\sqrt{\alpha} \hat{A}_{k} v^{i}\right)\right)+B\left(v^{i}\right) v^{i}-v_{k}^{(j)}\right]
\end{aligned}
$$

where $\beta$ is the first value in the sequence $1,1 / 2,1 / 4, \ldots, 2^{-n}$ for which the Armijo-Goldstein step principle[24]

$$
\left\|J\left(v^{i+1}\right)\right\|^{2} \leq\left\|J\left(v^{i}\right)\right\|^{2}-\frac{1}{2} \beta\left(\left\|\Delta v^{i}\right\|^{2}+\left\|R^{-1 / 2} B\left(v^{i}\right) \Delta v^{i}\right\|^{2}\right)
$$

is satisfied.

The iterative process ends when $\left\|v^{i+1}-v^{i}\right\|_{\infty} \leq \epsilon_{1}, J\left(v^{i}\right)-J\left(v^{i+1}\right) \leq \epsilon_{2} J\left(v^{i}\right)$ or the pre-set maximum number of iterations is exceeded $I_{\text {max }}$. In this work, $\epsilon_{1}=10^{-6}, \epsilon_{2}=10^{-4}$ and $I_{\text {max }}=10$.

Dealing with nonlinear observation functions in the context of Kalman filtering by using iterative schemes is not a new endeavor. The iterated Kalman filter (IKF) proposed by Jazwinski [11] follows a very similar algorithm, and was later interpreted as a Gauss-Newton scheme [25] and a Picard iteration [14]. Zupanski [12] was possibly the first researcher to propose a iterative scheme in the context of ensemble-based estimators. He proposed a variant of the ensemble transform Kalman filter (ETKF[26]) to minimize a cost function (or maximize the corresponding likelihood function) similar to Eq. 37. Gu et al. [13] later suggests an iterative Gauss-Newton update formula for the EnKF in which the observation function was linearized about each of the intermediate ensemble means. The major difference between the algorithm proposed here and the one of $\mathrm{Gu}$ et al. [13] is the fact that the linearization is done about the current estimate of the conditional mode for each of the ensemble members as opposed to using the ensemble mean. Convergence is usually obtained with 5 iterations or fewer, and the the the analysis step can be performed in parallel across the ensemble.

Another important detail is that when nonlinearities are present, there is no guarantee that the posterior remains a Gaussian and, therefore, the mode(s) and the mean may no longer coincide. The magnitude of this discrepancy, which may be considered yet another bias source, scales with the error variance and, therefore, is expected to decrease as the estimator converges.

\section{Numerical Experiments}

The dynamics of the flow are represented by the discretized 2-D incompressible Navier-Stokes equations, and the simulations were carried out using the Immersed Boundary Projection Method (IBPM) [27, 28] enhanced by the Lattice Green's Function (LGF) formulation [29, 30]. The LGF formulation enforces exactly the free-space boundary condition at infinity even though the computation domain is restricted to the region of non-zero vorticity near the immersed body. Time integration is performed using the standard Crank-Nicholson Adam-Bashfort second-order splitting method to avoid the stiffness introduced by the viscous term. The resulting scheme can be considered spatially second-order accurate everywhere except in the vicinities of the immersed body where the error is first-order. A nonlinear function maps the state of the system (vorticity field) to the force applied to each of the immersed body points, from which the normal and tangential stresses acting on the surface of the body can be evaluated.

The problem of interest is the flow past a flat plate at 30 degrees angle of attack and Reynolds number 200. The state of this system is represented by the vorticity at each of the grid points. With the purpose of analyzing the effects of resolution, meshes with grid Reynolds numbers $\left(\operatorname{Re}_{\Delta}=\operatorname{Re} \Delta x / c\right.$ where $\Delta x=\Delta y$ is the grid spacing) equal to 1,2 and 4 are used. The grid resolution for the estimator model will always correspond to the coarsest of the three meshes. As for the measurements, we sense the pressure at 10 equidistant locations over the plate every 0.05 convective time units.

The ensemble size should be large enough to be able to represent the most energetic subspace of the flow and any extra parameters added to the state vector. Since we have 35 bias parameters and previous studies[7] indicated that 24 ensemble members were enough to satisfactorily represent the flow statistics, the ensemble size was set to 60 . The initial ensemble is constructed using the improved sampling scheme proposed by Evensen [23]. First, a dataset of snapshots of the base solution spanning several vortex-shedding cycles is generated, from which the base mean flow $\bar{x}_{b}$ and the leading Proper Orthogonal Decomposition (POD) modes are computed. Then, the $q$ ensemble members are randomly sampled from the subspace spanned by the first $q$ POD modes of the data so that the ensemble average is $\bar{x}_{b}$ 
and the norm of ensemble covariance matrix matches the norm of the dataset covariance matrix. The relaxation-to-prior spread (RTPS - $\theta=0.9$ ) scheme of Whitaker and Hamill [21] is the multiplicative covariance inflation scheme chosen to mitigate the deleterious effects of a reduced ensemble size, as previous studies [7] showed that it outperforms the constant model of Anderson and Anderson [20].

In order to evaluate the performance of the estimator, the following metrics will be used:

- Estimate error: measures how the estimated state differs from the reference state:

$$
E_{x}=\frac{\left\|\bar{x}-x_{\text {ref }}\right\|}{\left\|x_{\text {ref }}\right\|} .
$$

- Estimate error: measures how the estimated state differs from the reference state:

$$
E_{y}=\frac{\left\|\bar{y}-y_{\text {ref }}\right\|}{\left\|y_{\text {ref }}\right\|} .
$$

- Ensemble state RMS: measures the spread of the ensemble:

$$
R M S_{x}=\sqrt{\frac{1}{q-1} \sum_{i=1}^{q} \frac{\left\|x_{i}-\bar{x}\right\|^{2}}{\|\bar{x}\|^{2}}}
$$

\section{A. Identification of the Grid-resolution Model Error}

The numerical error introduced by the different resolution levels is the source of the bias that we will be interested in tracking. State statistics are estimated from a dataset of the base solutions spanning a sufficiently long time window. Bias statistics can then be estimated using the definitions presented in sec. III

$$
\begin{aligned}
\Delta & =\left[\begin{array}{lll}
\Pi x_{2}^{f}-f\left(\Pi x_{1}^{f}\right) & \cdots & \Pi x_{n}^{f}-f\left(\Pi x_{n-1}^{f}\right)
\end{array}\right] \\
\bar{\delta} & =\frac{1}{n-1} \Delta \mathbb{1} \\
E & =\left[\begin{array}{llll}
\Pi h^{f}\left(x_{1}^{f}\right)-h^{c}\left(\Pi x_{1}^{f}\right) & \cdots & \Pi h^{f}\left(x_{n}^{f}\right)-h^{c}\left(\Pi x_{n-1}^{f}\right)
\end{array}\right] \\
\bar{\epsilon}^{r} & =\frac{1}{n-1} E \mathbb{1}
\end{aligned}
$$

where the superscripts $f$ and $c$ correspond, respectively, to the fine and coarse meshes, and $\Pi$ is the interpolation operator between the fine and coarse meshes.

Figure 1 shows the temporal mean of the bias fields of both the dynamics $(\delta)$ and observation model $\left(\epsilon^{r}\right)$ between the $R e_{\Delta}=4$ and $R e_{\Delta}=1$ resolution levels. The mean bias in the dynamics seems to concentrate near the body where the error introduced by the immersed boundary dominates. Regarding the observation model, most of the bias seems to be restricted to the leading and trailing edges region. Part of this error is due to the action of the interpolation operator, but part of it is due to the discretization error of the observation operator itself.

The structure of the corresponding state and observation bias covariance matrices can be analyzed by computing its Proper Orthogonal Decomposition (POD), which can be accomplished by computing the left singular vectors of $\Delta-\bar{\delta}$ and $E-\bar{\epsilon}^{\wedge} r$, respectively. The low-rank bias representation proposed in Sec. IV] is justified on the fact that most of the bias variance is restricted to just a few directions in the state space. As Fig. 2 indicates, retaining the first $n_{s}=25$ state POD modes and $n_{o}=10$ observation POD modes leaves less than $0.001 \%$ of the variance to be modeled as white noise.

Therefore, matrices $\Gamma_{x}, \Gamma_{y}, \bar{\xi}$ and $\bar{\eta}$ in Eqs. 8 and 9 can be defined as

$$
\begin{aligned}
\Gamma_{x} & =\left[\begin{array}{llll}
\bar{\delta} & u_{1}^{s} & \cdots & u_{n_{s}}^{s}
\end{array}\right] \\
\Gamma_{y} & =\left[\begin{array}{llll}
\bar{\epsilon}^{r} & u_{1}^{o} & \cdots & u_{n_{o}}^{o}
\end{array}\right] \\
\bar{\xi} & =\bar{\eta}=\left[\begin{array}{llll}
1 & 0 & \cdots & 0
\end{array}\right]^{T}
\end{aligned}
$$

where $u_{i}^{s}$ and $u_{i}^{o}$ are the $i$-th leading POD modes of of $\Delta-\bar{\delta}$ and $E-\bar{\epsilon}^{\wedge} r$, respectively, normalized by their respective variances. The auto-regressive model parameters are set to $\phi_{x}=\phi_{y}=e^{-\Delta t / \tau}$, where $\Delta t$ is the time interval between two analysis steps, and $\tau$ is some reference decorrelation time length, here considered to be the plate shedding period. 

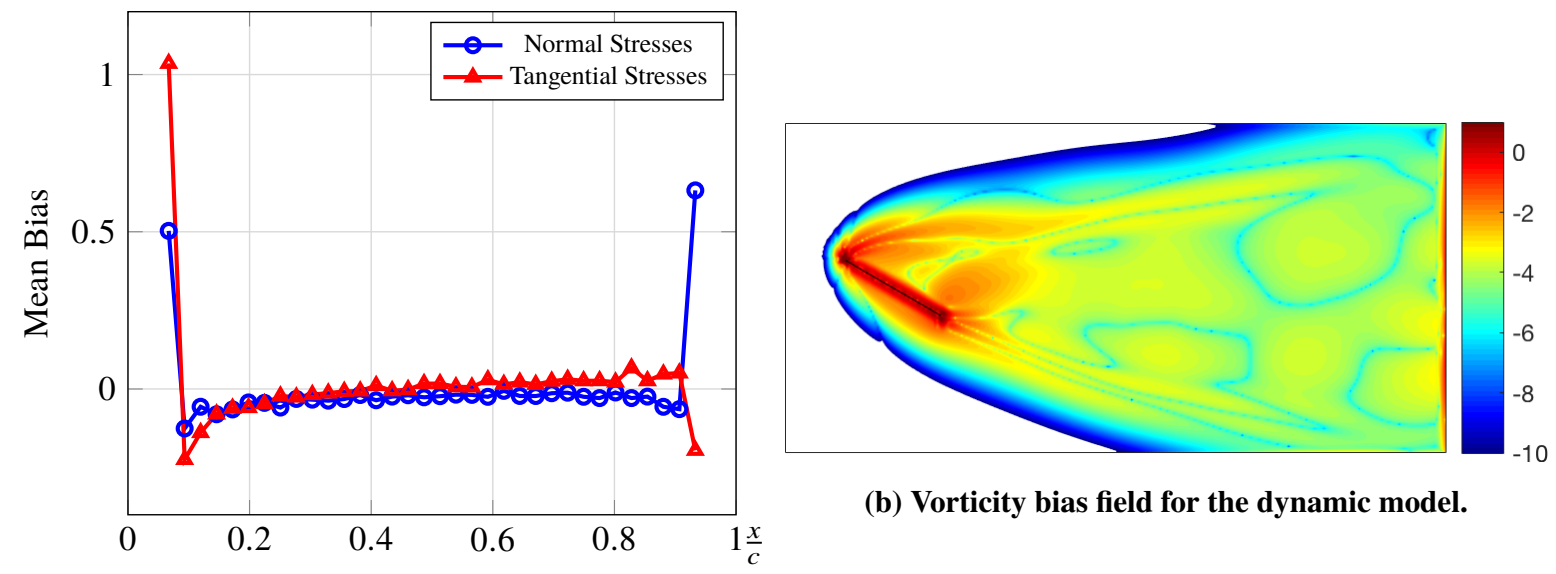

(b) Vorticity bias field for the dynamic model.

(a) Bias field for the observation model

Fig. 1 Temporal average of the bias fields introduced by the resolution error when comparing the $R e_{\Delta}=1$ (200 grid points per chord) simulation to the corresponding $R e_{\Delta}=4$ (50 grid points per chord) simulation.

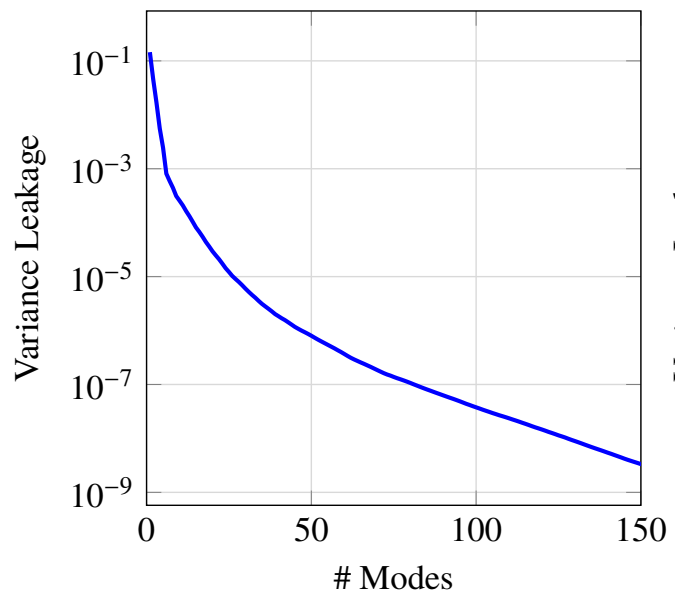

(a) State bias.

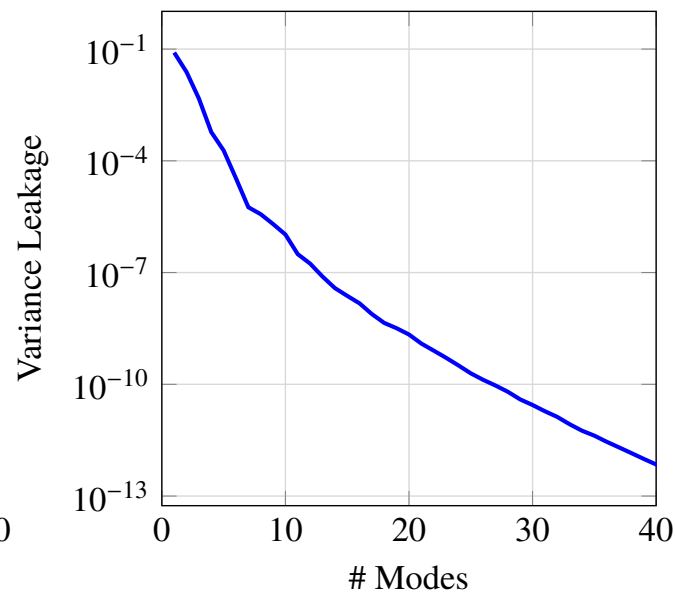

(b) Observation (pressure) bias.

Fig. 2 Fraction of the bias variance left out by using the corresponding first POD modes.

\section{B. Effect of nonlinearity in the measurement function}

We first analyze how the choice of the method to deal with the nonlinear observation function impacts the performance of the estimator in a perfect-model framework. Here, the reference solution was taken to be a simulation carried out in the same resolution level used by the estimator. Whenever the iterative method (Gauss-Newton) is used, the initial guess is obtained by the implicit linearization scheme. Since the maximum-likelihood estimate produced by the iterative method will only correspond to the minimum variance estimate in the case of a linear observation function, it is expected the posterior variance obtained by the iterative method will be larger than optimal. It was also noted that, at the initial assimilation steps, the iterative scheme achieves a reduction of the cost function of at least two orders of magnitude. As the estimation progresses and the state variance decreases, the magnitude of the corrections decreases and the cost improvement also becomes marginal (see Fig. 3a.

Despite its simplicity and lower cost, the implicit linearization scheme performs surprisingly well compared to the iterative method. Figure 3 a shows that this schemes performs better, or at least similarly, than the iterative scheme for all the studied measurement noise levels $(R)$. It is not clear how well the linearization about the ensemble mean is supposed to approximate the conditional mean (minimum error variance) of the posterior distribution, but results seem to indicate that it yield a better approximation than the iterative method. 
In the absence of nonlinearities and modeling errors, the terminal state error level is supposed to decrease as the measurement noise level decreases (see [7]). Since errors due to the presence of nonlinearities in the observation function are expected to scale with $h_{x x} \hat{C}_{k}^{x x}[11]$, and Fig. $3 \mathrm{~b}$ shows that lower $R$ consistently produces smaller variances, the saturation of the state error at $10^{-3}$ for both the schemes is unexpected. Further study is necessary to investigate what source of error, independent of $R$ or $\hat{C}_{k}$, is dominating the long term behavior of the filter.

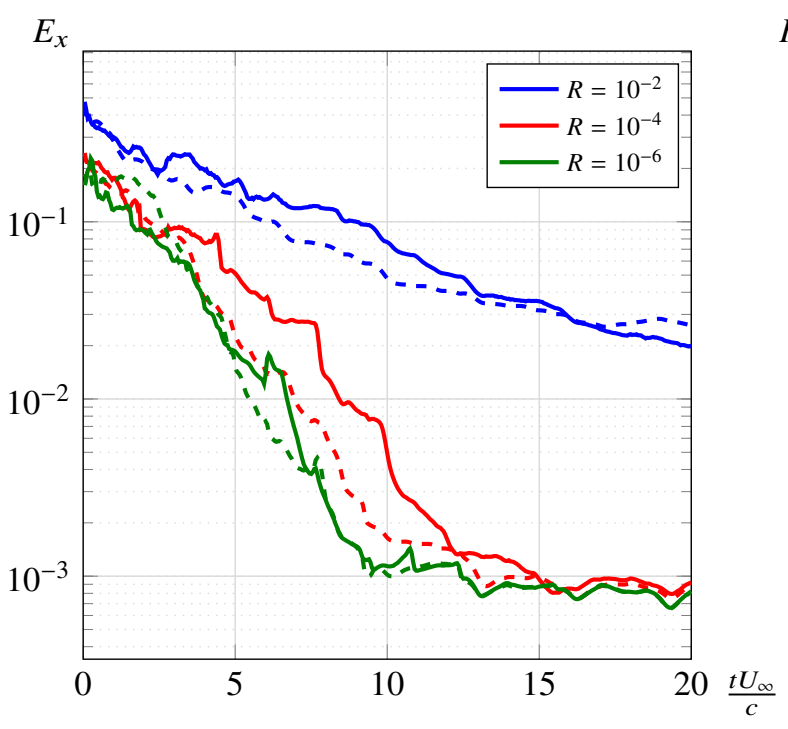

(a) State error evolution.

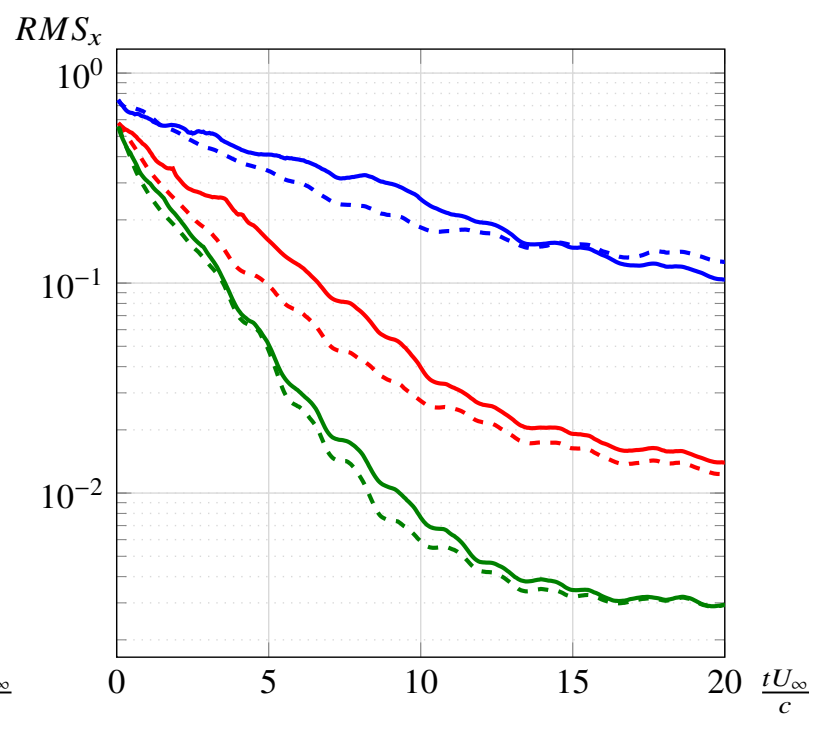

(b) Observation error evolution.

Fig. 3 Effect of nonlinear observation model in the estimator performance in the perfect-model framework. Solid lines correspond to the iterative (Gauss-Newton) method and dashed lines, to the implicit linearization scheme.

\section{Effect of the Bias}

In this section we consider the case when the low-resolution model is used to track the high-resolution data without an explicit treatment of the model biases. Since most of the bias in the system dynamics is restricted to the region close to the body (see Fig. 1), we introduce a second error metric that evaluates the mean-square error of the solution in the region outside the blue circle represented in Fig. 4 In this way we can distinguish estimator errors associated with forces on the immersed surface from those associated with the wake dynamics. The measurement error level is set to $R=10^{-4}$ for all simulation henceforward. Process noise can be added to the low-resolution dynamics to inform the estimator of a reduction of the perceived reliability of the dynamic model. Since it is presumed that no information about the bias is available, additive covariance inflation (which is equivalent to process noise in this context) will be sampled from the same subspace from which the initial ensemble was taken but their variance is scale by a factor $\lambda$.

Figure 5 compares the performance of the bias-blind estimator for different magnitudes of process noise. The base case (without process noise) is represented by the green curve. One can marginally improve this performance by adding the right amount of process noise (red curve). This will increase the perceived reliability of the measurements, increasing the responsiveness of the estimator and reducing the observation data mismatch. Note, however, that the average error, both close to the body (solid lines) and away from it (dashed lines) does not change significantly. Also, this approach has its limits: too much noise will eventually dominate the estimator dynamics (blue curve). That notwithstanding, the bias-blind estimator final error is two orders of magnitude larger than the one that could be achieved with in a perfect-model framework. 


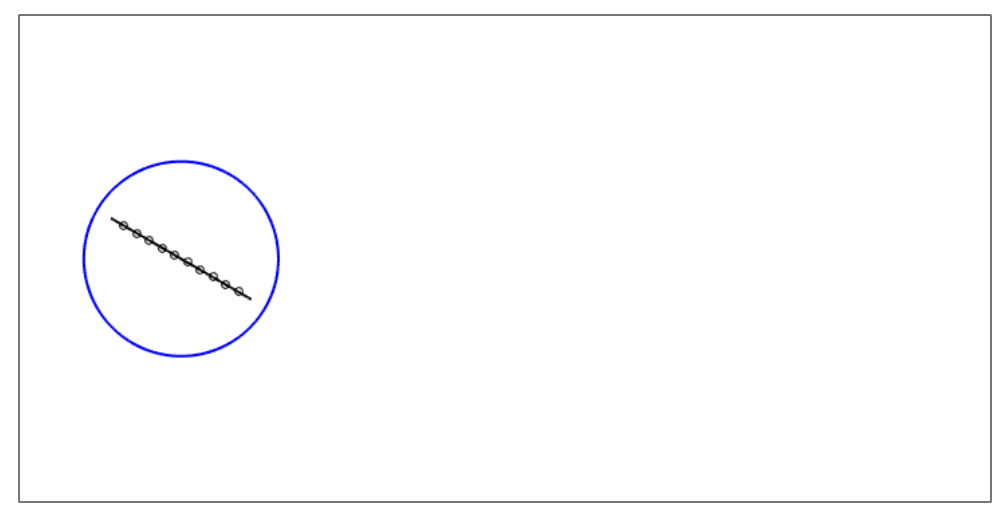

Fig. 4 Illustration of the different domains used for the evaluation of the state error. The small circles on the surface of the plate represents the locations where the pressure measurements are taken.

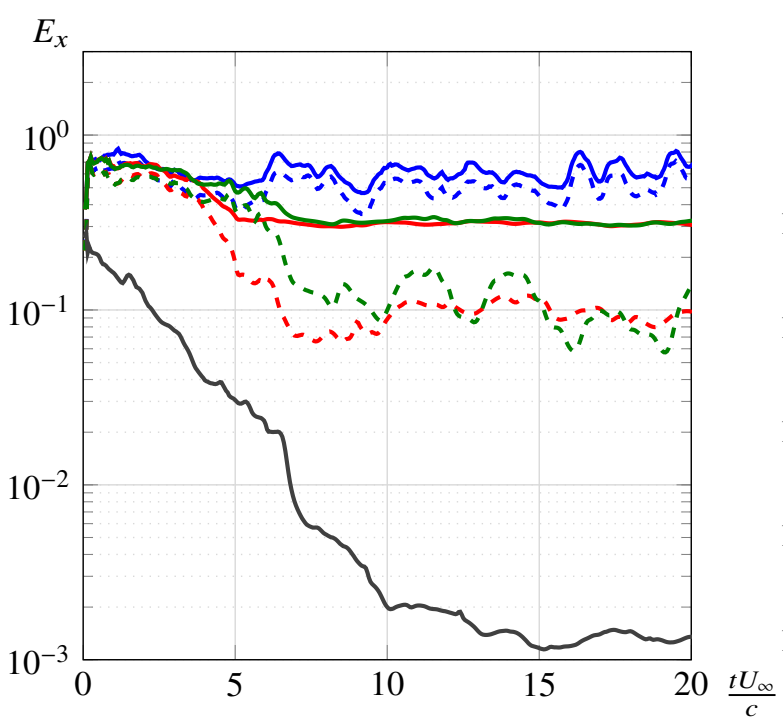

(a) State error evolution.

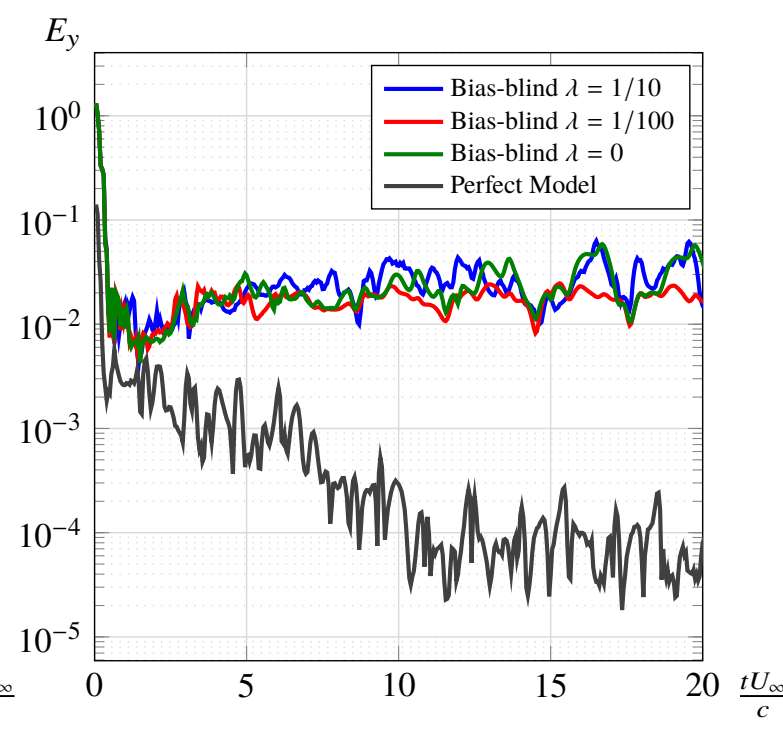

(b) Observation error evolution.

Fig. 5 Bias-blind estimator performance highlighting the deleterious effect of the dynamics and observation bias $\left(R=10^{-4}\right)$. Solid lines correspond to the mean square error evaluated in the entire computational domain, while the dashed lines restrict this evaluation to the region outside an unit circle centered at the plate.

\section{Bias-aware Estimation}

In this section we evaluate the proposed bias-aware estimator performance when the exact bias statistics are used to form both the $\Gamma$ matrices. Because the proposed scheme only adds $n_{o}+n_{s}=35$ degrees of freedom to the much larger state vector $x$ (about 15000 degrees of freedom), the additional computational cost is minimum. Figure 6a shows a 33\% state error improvement for the entire domain, while the error far from the body improves $60 \%$.

The bias dynamics are forced with process noise with covariance matrices $R^{b}=\lambda_{o} I_{n_{o}}$ and $Q^{b}=\lambda_{s} I_{n_{s}}$. The existence of process noise leads to a sustainably larger variance for the bias parameters, which allows for correction to be consistently made throughout the estimation window. This feature is especially important for problems like the present one, in which the bias cannot be considered slowly-varying. In fact, the bias is expected to exhibit a periodic behavior as the flow itself is periodic with the vortex-shedding period being the fundamental time scale. Figure 7 shows how different choices of the noise magnitudes impacts the state and observation error estimates. Larges values for the noise parameters favor smaller measurement mismatches (by allowing more aggressive analysis) at the expense of a possibly larger state error. 


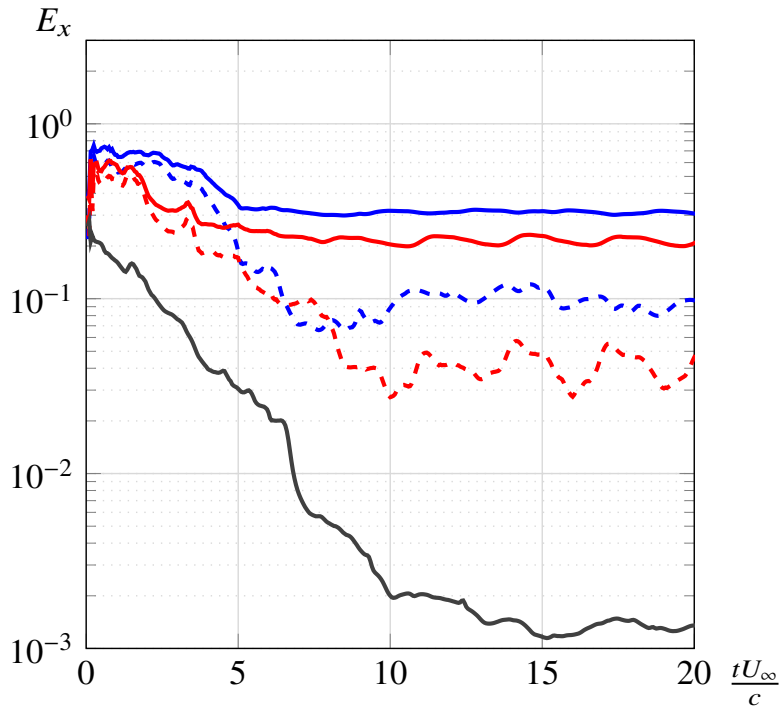

(a) State error evolution.

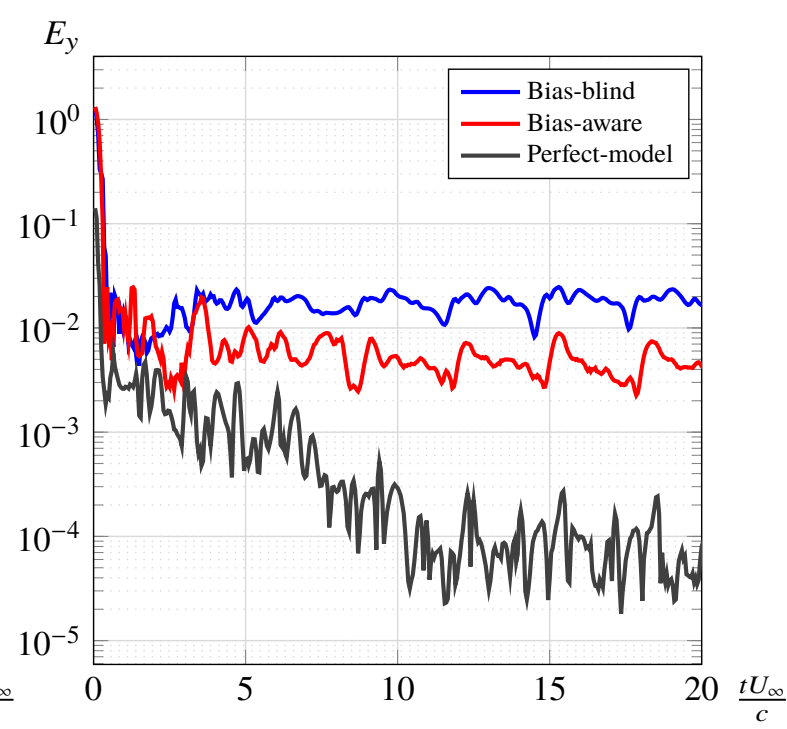

(b) Observation error evolution.

Fig. 6 Bias-aware estimator performance $\left(R=10^{-4}, \lambda_{o}=\sqrt{10} / 10\right.$ and $\left.\lambda_{s}=\sqrt{10} / 10\right)$. Solid lines correspond to the mean square error evaluated in the entire computational domain, while the dashed lines restrict this evaluation to the region outside an unit circle centered at the plate.

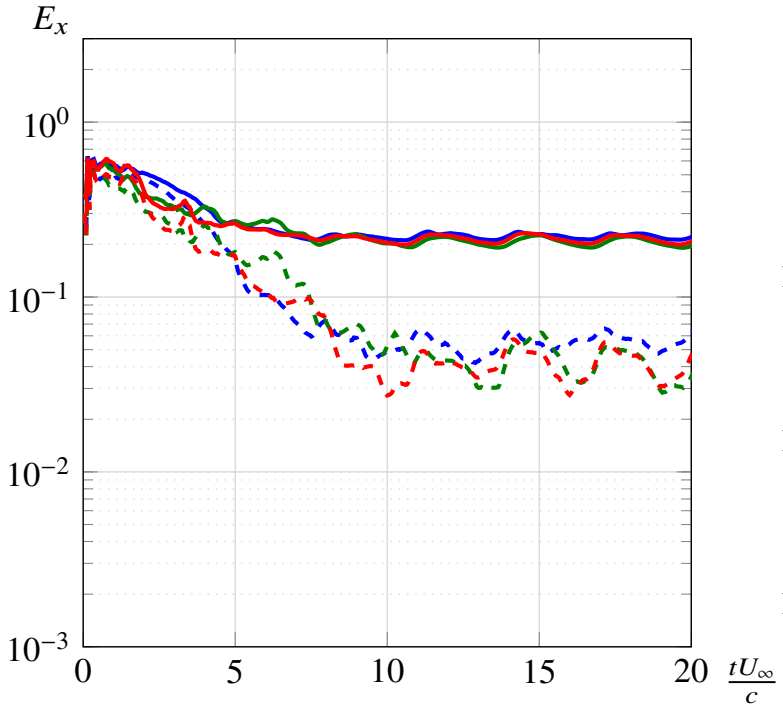

(a) State error evolution.

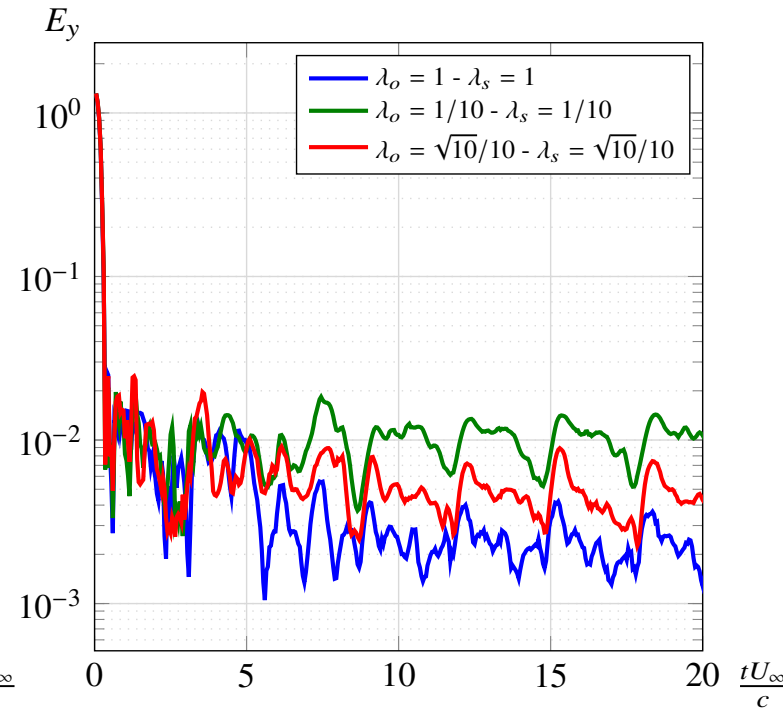

(b) Observation error evolution.

Fig. 7 Effect of the magnitude of the process noise to bias dynamics $\left(R=10^{-4}\right)$. Solid lines correspond to the mean square error evaluated in the entire computational domain, while the dashed lines restrict this evaluation to the region outside an unit circle centered at the plate.

As Fig. 6bindicates, bias correction decreases the pressure error by $80 \%$. Figure 8 displays an example of the correction introduced by the proposed scheme to the estimated output. Correction seems to be less effective near the leading edge, possibly because of the large pressure gradients that appear in there regions. As a consequence, global quantities like the lift coefficient also have their estimates improved. 


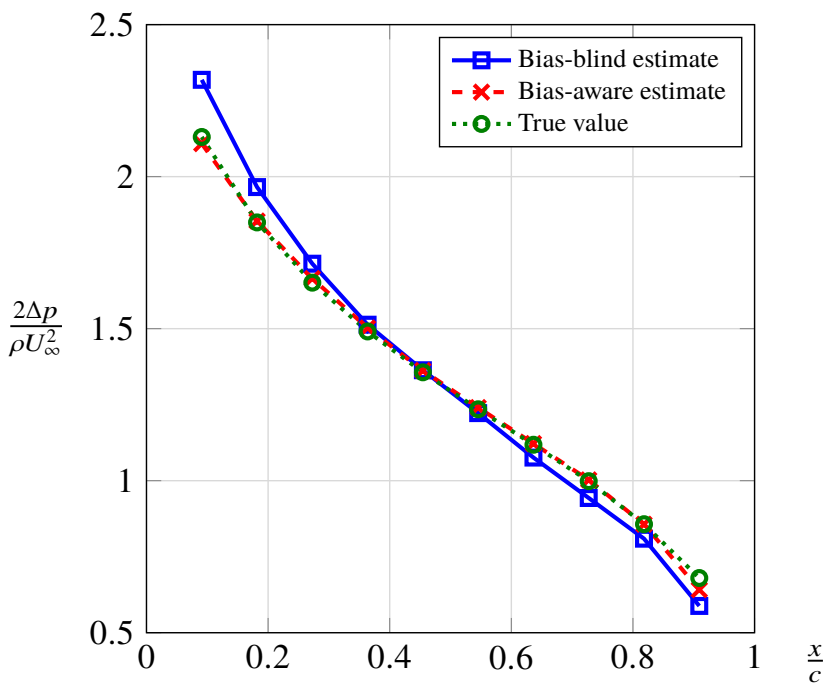

(a) Normal stresses.

Fig. 8 Estimated pressure on the surface of the flat plate at end of a simulation window $\left(t U_{\infty} / c=20\right)$.

\section{E. Imperfect Bias Statistics}

The biggest weakness of the proposed methodology is the need of a priori knowledge of the exact bias statistics, something that is rarely available. In order to show that good performance can also be achieved with imperfect statistics, an intermediary resolution $\left(R e_{\Delta}=2\right)$ is used in lieu of the fine-grid solution for the evaluation of the bias statistics. The performance of the resulting estimator (shown in Fig. 9p is very similar to the one obtained with the exact statistics. This seems to indicate that as long one can estimate the structure of the bias, explicitly tracking it is beneficial for the estimation.

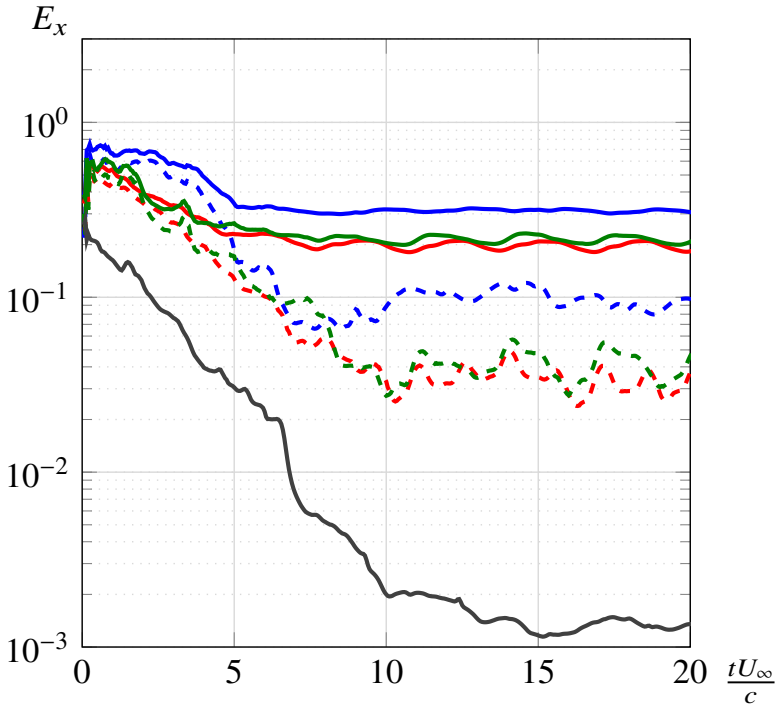

(a) State error evolution.

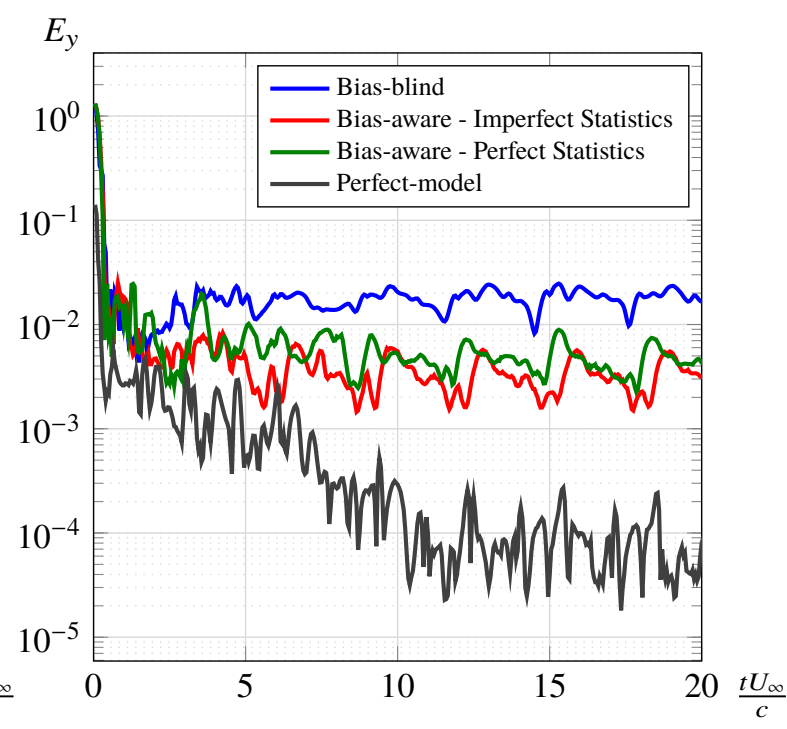

(b) Observation error evolution.

Fig. 9 Bias-aware estimator performance with imperfect statistics $\left(R=10^{-4}\right)$. Solid lines correspond to the mean square error evaluated in the entire computational domain, while the dashed lines restrict this evaluation to the region outside an unit circle centered at the plate. 


\section{Conclusions}

In this paper, we introduced a novel approach to use an EnKF framework to simultaneously mitigate the effects of biased forecast and observation models. In lieu of treating the bias as a single random variable, we split it into its slow and fast components. Colored noise processes are used to model the time correlation that the slow part of the bias is expected to exhibit, while fast component is modeled by white-noise processes. The restriction of the bias dynamics to the low-rank subspace that contains most of the variance allows for a more efficient sampling of the state space and enables the use of fewer ensemble members to satisfactorily represent the system statistics.

The performance of the proposed estimator is assessed by employing an ensemble of coarse-grid simulations to track a fine-grid simulation of the low-Re flow past a flat plate at high angle of attack. Measurement data consists of pressure at ten different locations on the surface of the flat plate. Because the pressure is a nonlinear function of the vorticity field, the effects of nonlinearities on the analysis step was discussed. Results indicated that, at least for the problem in question, Evensen's implicit linearization scheme outperforms iterative schemes that approximate the posterior conditional mean by the conditional mode. The bias-aware estimator obtained about $70 \%$ state and observation error reduction for a marginal cost increment. Similar performance was achieved even when imperfect bias statistics where used.

\section{Appendix A - Implicit Linearization}

The scheme proposed by Evensen [23] to deal with nonlinear observation functions can be understood as an approximate linearization about the ensemble-averaged state. To see this, notice that each of the columns of $L \tilde{G} \hat{A}_{k}$ is given by

$$
\begin{aligned}
\sqrt{q-1}\left\{L \tilde{G} \hat{A}_{k}^{w}\right\}_{j} & =h^{\dagger}\left(G \hat{z}_{k}^{(j)}\right)-\frac{1}{q} \sum_{i=1}^{q} h^{\dagger}\left(G \hat{z}_{k}^{(i)}\right) \\
& =h^{\dagger}\left(G \hat{z}_{k}^{(j)}\right)-\frac{1}{q} \sum_{i=1}^{q}\left[h^{\dagger}\left(G \overline{\hat{z}}_{k}\right)+\frac{\partial h^{\dagger}}{\partial z}\left(G \overline{\hat{z}}_{k}\right) G\left(\hat{z}_{k}^{(j)}-\overline{\hat{z}}_{k}\right)+O\left[\left(\hat{z}_{k}-\overline{\hat{z}}_{k}\right)^{2}\right]\right] \\
& =h^{\dagger}\left(G \hat{z}_{k}^{(j)}\right)-h^{\dagger}\left(G \overline{\hat{z}}_{k}\right)+O\left[\left(\hat{z}_{k}-\overline{\hat{z}}_{k}\right)^{2}\right] \\
& =\frac{\partial h^{\dagger}}{\partial z}\left(G \overline{\hat{z}}_{k}\right) G\left(\hat{z}_{k}^{(j)}-\overline{\hat{z}}_{k}\right)+O\left[\left(\hat{z}_{k}-\overline{\hat{z}}_{k}\right)^{2}\right]
\end{aligned}
$$

and, therefore,

$$
\begin{aligned}
\hat{C}_{k}^{y y} & =\frac{1}{q-1} \sum_{j=1}^{q}\left(h^{\dagger}\left(G \hat{z}_{k}^{(j)}\right)-\frac{1}{q} \sum_{i=1}^{q} h^{\dagger}\left(G \hat{z}_{k}^{(i)}\right)\right)\left(h^{\dagger}\left(G \hat{z}_{k}^{(j)}\right)-\frac{1}{q} \sum_{i=1}^{q} h^{\dagger}\left(G \hat{z}_{k}^{(i)}\right)\right)^{T} \\
& =\left[\frac{\partial h^{\dagger}}{\partial z}\left(G \overline{\hat{z}}_{k}\right)\right] G\left(\frac{1}{q-1} \sum_{j=1}^{q}\left(\hat{z}_{k}^{(j)}-\overline{\hat{z}}_{k}\right)\left(\hat{z}_{k}^{(j)}-\overline{\hat{z}}_{k}\right)^{T}\right) G^{T}\left[\frac{\partial h^{\dagger}}{\partial z}\left(G \overline{\hat{z}}_{k}\right)\right]^{T}+O\left[\left(\hat{z}_{k}-\overline{\hat{z}}_{k}\right)^{3}\right] \\
& =\left[\frac{\partial h^{\dagger}}{\partial z}\left(G \bar{z}_{k}\right)\right] G \hat{C}_{k}^{z z} G^{T}\left[\frac{\partial h^{\dagger}}{\partial z}\left(G \overline{\hat{z}}_{k}\right)\right]^{T}+O\left[\left(\hat{z}_{k}-\overline{\hat{z}}_{k}\right)^{3}\right]
\end{aligned}
$$

and

$$
\hat{C}_{k}^{z y}=\hat{C}_{k}^{z z} G^{T}\left[\frac{\partial h^{\dagger}}{\partial z}\left(\overline{\hat{z}}_{k}\right)\right]^{T}+O\left[\left(\hat{z}_{k}-\overline{\hat{z}}_{k}\right)^{3}\right]
$$

These approximations are consistent with the approximate minimization of an effective cost function

$$
J(z)=\frac{1}{2 \alpha}\left\|\tilde{G}^{-1} z-\hat{z}_{k}^{(j)}\right\|_{\hat{C} z z}^{2}+\frac{1}{2}\left\|y_{k}-h^{\dagger}(G \overline{\hat{z}})-\left[\frac{\partial h^{\dagger}}{\partial z}(G \overline{\hat{z}})\right](z-G \overline{\hat{z}})-v_{k}^{(j)}\right\|_{R}^{2}
$$

which corresponds to the cost function of an extended Kalman filter in which the linearization is performed about the ensemble average instead of the prior state of the particle. 


\section{Acknowledgments}

This work has been supported in part by a grant from AFOSR (FA9550-14-1-0328) with Dr. Douglas Smith as program manager. A.F.C. da Silva would like to thank the Ministry of Education of Brazil (Capes Foundation) for its support through a Science without Borders scholarship (Grant number BEX 12966/13-4). The authors also acknowledge Prof. David Williams (Illinois Institute of Technology), Prof. Jeff Eldredge (University of California, Los Angeles) and Prof. Andrew Stuart (California Institute of Technology) for helpful discussions of this work.

\section{References}

[1] Ahuja, S., and Rowley, C. W., "Feedback Control of Unstable Steady States of Flow Past a Flat Plate Using Reduced-Order Estimators," Journal of Fluid Mechanics, Vol. 645, 2010, p. 447. doi:10.1017/S0022112009992655.

[2] Flinois, T. L. B., and Morgans, A. S., "Feedback Control of Unstable Flows: A Direct Modelling Approach Using the Eigensystem Realisation Algorithm,” Journal of Fluid Mechanics, Vol. 793, 2016, p. 41. doi:10.1017/jfm.2016.111.

[3] Colburn, C. H., Cessna, J. B., and Bewley, T. R., "State Estimation in Wall-Bounded Flow Systems. Part 3. the Ensemble Kalman Filter," Journal of Fluid Mechanics, Vol. 682, 2011, pp. 289-303. doi:10.1017/jfm.2011.222.

[4] Kikuchi, R., Misaka, T., and Obayashi, S., "Assessment of Probability Density Function Based on POD Reduced-Order Model for Ensemble-Based Data Assimilation," Fluid Dynamics Research, Vol. 47, No. 5, 2015, p. 051403. doi:10.1088/0169$5983 / 47 / 5 / 051403$.

[5] Kato, H., Yoshizawa, A., Ueno, G., and Obayashi, S., "A Data Assimilation Methodology for Reconstructing Turbulent Flows Around Aircraft,” Journal of Computational Physics, Vol. 283, No. C, 2015, pp. 559-581. doi:10.1016/j.jcp.2014.12.013.

[6] Mons, V., Chassaing, J.-C., Gomez, T., and Sagaut, P., "Reconstruction of Unsteady Viscous Flows Using Data Assimilation Schemes," Journal of Computational Physics, Vol. 316, 2016, pp. 255 - 280. doi:10.1016/j.jcp.2016.04.022.

[7] da Silva, A. F. d. C., and Colonius, T., "An EnKF-based Flow State Estimator for Aerodynamic Flows," 8th AIAA Theoretical Fluid Mechanics Conference, American Institute of Aeronautics and Astronautics, Reston, Virginia, 2017. doi:10.2514/6.2017-3483.

[8] Friedland, B., "Treatment of bias in recursive filtering," IEEE Transactions on Automatic Control, Vol. 14, No. 4, 1969, pp. 359-367. doi:10.1109/TAC.1969.1099223.

[9] Dee, D. P., and Da Silva, A. M., "Data assimilation in the presence of forecast bias," Quarterly Journal of the Royal Meteorological Society, Vol. 124, No. 545, 1998, pp. 269-295. doi:10.1002/qj.49712454512.

[10] Drecourt, J.-P., Madsen, H., and Rosbjerg, D., "Bias Aware Kalman Filters: Comparison and Improvements," Advances in Water Resources, Vol. 29, No. 5, 2006, pp. 707 - 718. doi:10.1016/j.advwatres.2005.07.006.

[11] Jazwinski, A. H., Stochastic processes and filtering theory, Academic Press, Inc., 1970.

[12] Zupanski, M., "Maximum Likelihood Ensemble Filter: Theoretical Aspects," Monthly Weather Review, Vol. 133, No. 6, 2005, pp. 1710-1726. doi:10.1175/MWR2946.1.

[13] Gu, Y., Oliver, D. S., et al., "An iterative ensemble Kalman filter for multiphase fluid flow data assimilation," SPE Journal, Vol. 12, No. 04, 2007, pp. 438-446. doi:10.2118/108438-PA.

[14] Cohn, S. E., "An Introduction to Estimation Theory," Journal of the Meteorological Society of Japan. Ser. II, Vol. 75, No. 1B, 1997, pp. 257-288. doi:10.2151/jmsj1965.75.1B_257.

[15] Dee, D. P., "On-line Estimation of Error Covariance Parameters for Atmospheric Data Assimilation," Monthly Weather Review, Vol. 123, No. 4, 1995, pp. 1128-1145. doi:10.1175/1520-0493(1995)123<1128:OLEOEC>2.0.CO;2.

[16] Chui, C. K., and Chen, G., Kalman Filtering With Real Time Applications, Springer, 2009. doi:10.1007/978-3-319-47612-4.

[17] Evensen, G., "Sequential Data Assimilation With a Nonlinear Quasi-Geostrophic Model Using Monte Carlo Methods to Forecast Error Statistics," Journal of Geophysical Research: Oceans, Vol. 99, No. C5, 1994, pp. 10143-10162. doi:10.1029/94JC0057.

[18] Julier, S. J., and Uhlmann, J. K., “Unscented Filtering and Nonlinear Estimation,” Proceedings of the IEEE, Vol. 92, No. 3, 2004, pp. 401-422. doi:10.1109/JPROC.2003.823141.

[19] van Leeuwen, P. J., "Comment on "Data Assimilation Using an Ensemble Kalman Filter Technique"," Monthly Weather Review, Vol. 127, No. 6, 1999, pp. 1374-1377. doi:10.1175/1520-0493(1999)127<1374:CODAUA>2.0.CO;2. 
[20] Anderson, J. L., and Anderson, S. L., "A Monte Carlo Implementation of the Nonlinear Filtering Problem to Produce Ensemble Assimilations and Forecasts," Monthly Weather Review, Vol. 127, No. 12, 1999, pp. 2741-2758. doi:10.1175/15200493(1999)127<2741:AMCIOT>2.0.CO;2.

[21] Whitaker, J. S., and Hamill, T. M., "Evaluating Methods to Account for System Errors in Ensemble Data Assimilation," Monthly Weather Review, Vol. 140, No. 9, 2012, pp. 3078-3089. doi:10.1175/MWR-D-11-00276.1.

[22] Evensen, G., and Van Leeuwen, P. J., "Assimilation of Geosat Altimeter Data for the Agulhas Current Using the Ensemble Kalman Filter With a Quasigeostrophic Model," Monthly Weather Review, Vol. 124, No. 1, 1996, pp. 85-96. doi:10.1175/15200493(1996)124<0085:AOGADF>2.0.CO;2.

[23] Evensen, G., Data Assimilation: The Ensemble Kalman Filter, Springer Science \& Business Media, 2009.

[24] Bjorck, A., 9. Nonlinear Least Squares Problems, 1996, Chap. 9, pp. 339-358. doi:10.1137/1.9781611971484.ch9.

[25] Bell, B. M., and Cathey, F. W., "The iterated Kalman filter update as a Gauss-Newton method," IEEE Transactions on Automatic Control, Vol. 38, No. 2, 1993, pp. 294-297. doi:10.1109/9.250476.

[26] Bishop, C. H., Etherton, B. J., and Majumdar, S. J., "Adaptive Sampling with the Ensemble Transform Kalman Filter. Part I: Theoretical Aspects," Monthly Weather Review, Vol. 129, No. 3, 2001, pp. 420-436. doi:10.1175/1520-0493(2001)129<0420: ASWTET>2.0.CO;2.

[27] Taira, K., and Colonius, T., “The Immersed Boundary Method: A Projection Approach,” Journal of Computational Physics, Vol. 225, No. 2, 2007, pp. 2118-2137. doi:10.1016/j.jcp.2007.03.005.

[28] Colonius, T., and Taira, K., "A Fast Immersed Boundary Method Using a Nullspace Approach and Multi-Domain Far-Field Boundary Conditions," Computer Methods in Applied Mechanics and Engineering, Vol. 197, No. 25, 2008, pp. 2131-2146. doi:10.1016/j.cma.2007.08.014.

[29] Liska, S., and Colonius, T., "A Parallel Fast Multipole Method for Elliptic Difference Equations," Journal of Computational Physics, Vol. 278, 2014, pp. 76-91. doi:10.1016/j.jcp.2014.07.048.

[30] Liska, S., and Colonius, T., "A Fast Immersed Boundary Method for External Incompressible Viscous Flows Using Lattice Green's Functions,” Journal of Computational Physics, Vol. 331, 2017, pp. 257-279. doi:10.1016/j.jcp.2016.11.034. 